\title{
Difficulties with Multiple Time Stepping and Fast Multipole Algorithm in Molecular Dynamics
}

\author{
THOMAS C. BISHOP, ROBERT D. SKEEL, KLAUS SCHULTEN \\ Beckman Institute, 405 North Mathews Street, University of Illinois at Urbana-Champaign, Urbana, \\ Illinois 61801
}

Received 17 December 1996; accepted 19 May 1997

\begin{abstract}
Numerical experiments are performed on a 36,000-atom protein-DNA-water simulation to ascertain the effectiveness of two devices for reducing the time spent computing long-range electrostatics interactions. It is shown for Verlet-I/r-RESPA multiple time stepping, which is based on approximating long-range forces as widely separated impulses, that a long time step of $5 \mathrm{fs}$ results in a dramatic energy drift and that this is reduced by using an even larger long time step. It is also shown that the use of as many as six terms in a fast multipole algorithm approximation to long-range electrostatics still fails to prevent significant energy drift even though four digits of accuracy is obtained. (c) 1997 John Wiley \& Sons, Inc. J Comput Chem 18: 1785-1791, 1997
\end{abstract}

Keywords: multiple time steps; r-RESPA; Verlet method; molecular dynamics; fast multipole method

\section{Introduction}

$\mathbf{T}$ he computation of full electrostatics has been avoided in molecular dynamics (MD) simulations because of the high cost of doing the direct

Correspondence to: R. D. Skeel

Contract/grant sponsor: NSF; contract/grant numbers BIR9318159 and DMS-960088

Contract/grant sponsor: NIH; contract/grant number P41RR05969 calculation. Nonetheless, it is generally recognized that neglect of long-range electrostatics introduces serious artifacts in many situations. ${ }^{1-6}$ This is due to the slow decay of long-range electrostatics effects with distance as illustrated in Figure 1 for an approximately 36,000 -atom solvated protein-DNA system studied in the literature. ${ }^{7,8}$ Fortunately, in recent years two computational techniques have been developed that make the cost of full electrostatics more manageable: multiple time step (MTS) integrators reduce the frequency with which longrange forces need to be computed and innovative 


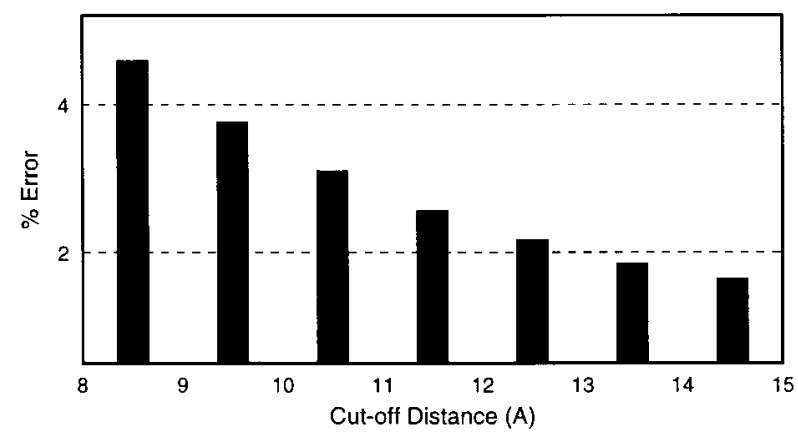

FIGURE 1. Error in the electrostatic energy due to the use of cutoff approximations for a protein-DNA - waterion system.

fast electrostatics algorithms reduce the cost of calculating pairwise electrostatic forces for $N$ particles from order $N^{2}$ to order $N \log N$, or to order $N$ in the case of the fast multipole algorithm (FMA) applied to nonperiodic systems. MTS methods and the FMA are both implemented in the biomolecular modeling system MDScope ${ }^{9}$ in its simulation program NAMD, ${ }^{10}$ which was built from scratch using an object-oriented design to achieve high performance on parallel computers. In this article NAMD is applied to a realistic molecular system to study the limits of MTS methods and the FMA. Results of the computer experiments suggest that: the highest frequency present in the force field imposes a limit of slightly less than half its period on the longest time step that can be used; and the discontinuities inherent in tree algorithms such as the FMA cause instability unless the forces are computed very accurately, for example, by using a large number of terms in a multipole expansion.

Attempts to exploit the multiple time scale character of the $N$-body problem by using MTS (different time steps for different interactions) date back to 1967 in astrophysics ${ }^{11}$ and to 1978 in MD. ${ }^{12}$ A simple version of such a technique was implemented in the program GROMOS where it is called a twin-range method. ${ }^{13}$ The long-range forces were calculated typically every 10 time steps and then held constant. More accurate is the local reaction field method, ${ }^{14}$ which calculates the first four Taylor series terms of the long-range potential at the center of each "charge group" every $m$ time steps and uses this to evaluate the long-range force on each atom of the charge group at every time step. Another advance in formulas for MTS is the 1989-1991 work, ${ }^{3,15}$ which introduces formulas based on the idea of "Verlet equivalence." The idea is that if the short-range forces are not pre- sent, then the integration of the long-range forces should reduce to that of the Verlet method with long time steps, thus obtaining to some extent the favorable properties of the Verlet method (reversibility, symplecticness, second-order accuracy) for all time scales. Notable among these schemes is the one called Verlet-I, which effectively amounts to introducing the long-range force as an impulse whenever it is evaluated. However, the impulse method is not pursued in ref. 3 because of an awareness that it was subject to resonance if the long time step $\Delta t$ happened to coincide with a natural frequency of the system, which could lead to erroneous forces. A more abstract derivation of the impulse MTS method based on operator splitting appeared in 1992 under the name r-RESPA. ${ }^{16}$ Subsequent work $^{17}$ showed that the method retained the symplectic property ${ }^{18}$ of Verlet and it experimentally verified for simple test problems the seriousness of the resonance. The use of individual time steps that are fixed for the duration of the simulation is adequate for bonded interactions. However, it is not adequate for nonbonded interactions because of a need to vary the size of the time step as the distance changes. This variation can be done without sacrificing properties such as reversibility, symplecticness, and second-order accuracy by using a switching function to artificially break up the interaction potential into a fast and a slow part. ${ }^{16,19}$ More benefit from MTS methods is attained by employing not just two but several different time steps; for example, eight are used in ref. 3, four in refs. 20 and 21, and five in ref. 22. The biggest gain, of course, occurs in going from one time step to two, and we focus our investigation on this special case.

A recent innovation of importance of MD is the construction of order $N \log N$ and order $N$ algorithms for fast electrostatics, such as the fast multipole method, ${ }^{23,24}$ which improves the earlier work on tree algorithms. ${ }^{25,26}$ Initially, this generated expectations that full electrostatics could be achieved at low cost. The most optimistic statement in this regard is an early version of promotional material ${ }^{27}$ for the high performance computing initiative, which has a graph showing that the fast multipole method gives an $N$-fold improvement over existing algorithms. This, of course, ignores the hidden constant in the $O(N)$ estimate. An early implementation $^{28}$ yielded instead a speedup of $N / 10,000$, which was cited in ref. 29 as a reason not to include full electrostatics. Recently more efficient implementations have become available; for example, the enhanced FMA described in ref. 
30, and yet faster versions of the fast multipole method are promised. ${ }^{31}$

The combination of fast electrostatics with MTS is first reported in the literature in ref. 32 for the simple constant force MTS method and in ref. 22 for the impulse MTS method. The constant force method demonstrated in ref. 22 does not conserve energy very well at all. The program NAMD incorporates an implementation of an enhanced FMA called DMPTA ${ }^{33}$ and implements various MTS integrators, including the impulse method. The combination of an impulse MTS integrator with Ewald sums for periodic boundary conditions is described in ref. 21.

\section{Difficulties with MTS}

In the simples case, MD is modeled by Newton's second law of motion,

$$
M \ddot{x}=F(x),
$$

where $M$ is a diagonal matrix of masses, $x$ is the collection of all particle positions, and $F$ is the collection of forces obtained as the gradient of an empirical potential energy function. This function is a sum of a very large number of 2-, 3-, and 4-body interactions, which (after taking masses into account) have a fairly large range of time scales associated with them. When MTS integration is performed, slower forces are computed less frequently than faster forces. The Verlet-I/ r-RESPA impulse algorithm is described below in the case where the potential energy is split into two parts, yielding $F=F_{\text {fast }}+F_{\text {slow }}$, and two time steps $\delta t$ and $\Delta t=m \delta t$ are used:

$$
\left.\begin{array}{l}
V_{n, 0}=V_{n}+(\Delta t / 2) M^{-1} F_{\text {slow }}\left(X_{n}\right), \\
X_{n, 0}=X_{n} \\
V_{n, k+1 / 2}=V_{n, k}+(\delta t / 2) M^{-1} F_{\text {fast }}\left(X_{n, k}\right) \\
X_{n, k+1}=X_{n, k}+\delta t V_{n, k+1 / 2} \\
V_{n, k+1}=V_{n, k+1 / 2}+(\delta t / 2) M^{-1} F_{\text {fast }}\left(X_{n, k+1}\right)
\end{array}\right\}
$$

Typical time steps $\delta t$ for MD are $0.5-1 \mathrm{fs}$. Much larger values might be expected for $\Delta t$. For exam- ple, computational experiments ${ }^{34}$ show a $98 \%$ correlation in long-range forces after $16 \mathrm{fs}$.

However, as explained in the Introduction, there is a problem with resonance for the impulse MTS method. This can be seen by recognizing the method as simply the analytical solution of

$$
\begin{aligned}
M \ddot{X}= & \sum_{k=-\infty}^{+\infty} \delta t \delta(t-k \delta t) F_{\text {fast }}(X) \\
& +\sum_{n=-\infty}^{+\infty} \Delta t \delta(t-n \Delta t) F_{\text {slow }}(X) .
\end{aligned}
$$

The point is clearer if we assume that the substep $\delta t$ is sufficiently small to allow the following approximation to the preceding equation:

$$
M \ddot{X}=F_{\text {fast }}(X)+\sum_{n=-\infty}^{+\infty} \Delta t \boldsymbol{\delta}(t-n \Delta t) F_{\text {slow }}(X) .
$$

This equation, which was first published in ref. 35, makes apparent the possibility that the periodic pulses of slow forces will resonate with a natural frequency of the fast forces. (In the case of the actual finite- $\delta \mathrm{t}$ simulation the frequencies of the fastest forces will be slightly increased due to the "blue shift" caused by the Verlet method.) In MD the vibrations of covalently bonded hydrogen atoms have periods from 9 to $10 \mathrm{fs}$, so instability would be expected if the long time step is in that range. This is apparent in the results of ref. 34 . Also, extensive MD experiments with Langevin damping show the shortcomings of the impulse method with long time steps. ${ }^{36,37}$

Other researchers obtain satisfactory results with the impulse MTS method only for time steps much less than $9-10$ fs. For example, satisfactory results are obtained in ref. 20 for long time steps of only 3 or $4 \mathrm{fs}$ with markedly worse results for $5 \mathrm{fs}$. In figure 3 of ref. 22 good energy conservation is obtained for long time steps only up to $4.5 \mathrm{fs}$, and there is pronounced degradation in going from 4 to $4.5 \mathrm{fs}$.

Similar experiences were obtained from the use of NAMD to simulate a 36,573-atom proteinDNA-water-ion system. Of these atoms nearly $90 \%$ constitute 11,040 flexible TIP3P water molecules. Figure 2 shows energy as a function of time for an implementation of the impulse two time step method for a short time step $\delta t=1 \mathrm{fs}$ and various long time steps $\Delta t$. The slow force includes only the part of the electrostatic force left 

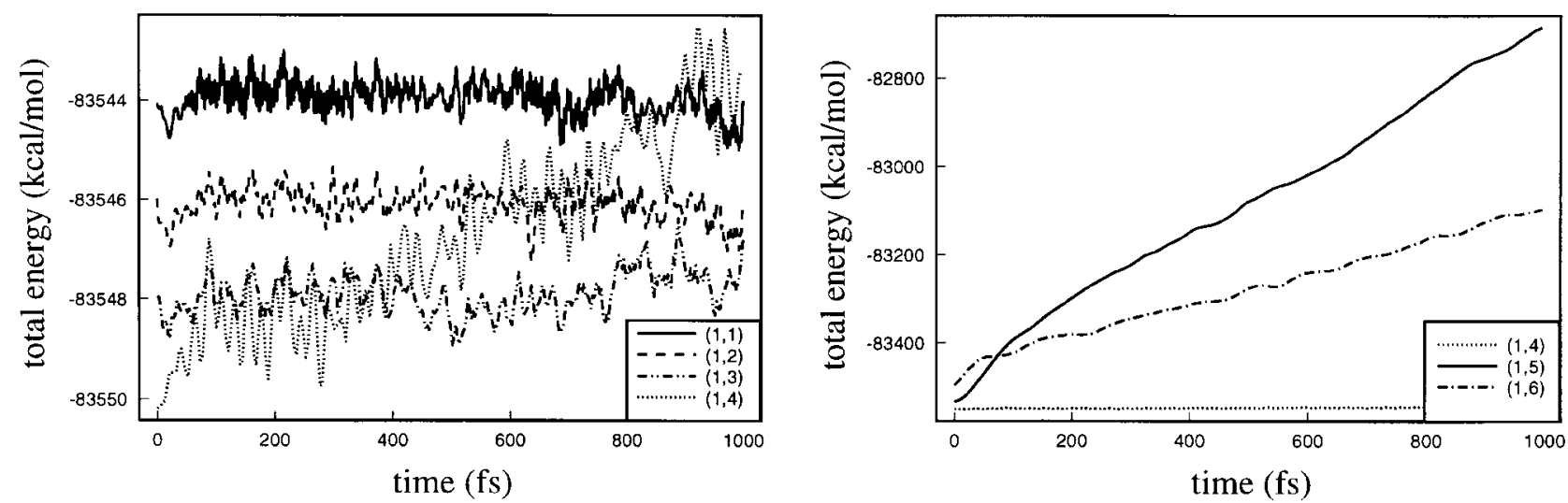

FIGURE 2. Conservation of energy $(\mathrm{kcal} / \mathrm{mol})$ as a function of time $(\mathrm{fs})$ for a variety of long time steps for the inpulse multiple time step scheme. The short and long time steps are indicated in femtoseconds as (short, long) in each graph. In the graph on the left the "base energy" of each plot is shifted to separate the plots from each other.

over after subtracting a short-range electrostatic force. A switching function given in ref. 20 is used to effect a smooth transition between a short-range (fast) force at $7.0 \AA$ and a long-range (slow) force at $8.5 \AA$. (Any electrostatic interaction at a distance less than $8.5 \AA$ has a time step of $1 \mathrm{fs}$ and anything greater has a time step of $j \mathrm{fs}, j=1,2,3,4,5,6$.)

The first graph shows noticeable energy drift for a long time step of $4 \mathrm{fs}$ and the second graph demonstrates that this becomes dramatic at $5 \mathrm{fs}$ but then decreases at $6 \mathrm{fs}$ - an indication of a resonancelike phenomenon. A check of the different contributions to the total energy indicates that the energy gain occurred via the bonded energies. These observations suggest difficulties associated with values of $\Delta t$ at nearly half the period of the highest frequency modes in the system, which are vibrations of hydrogen atoms.

These difficulties cannot be attributed to ordinary resonance because they occur not at the period of a natural frequency but near half its period. An intricate analysis of a linear 1-dimensional 2 degree of freedom system ${ }^{38}$ explains this as a numerical instability inherent in the impulse MTS method. A purpose of the present article is to demonstrate the relevance of this instability for MD.

The maximum value of $\Delta t$ consistent with stability is undoubtedly affected by both the choice of the smaller time step $\delta t$ and the splitting between $F_{\text {fast }}$ and $F_{\text {slow }}$. For example, Fig. 3 shows the results of a simulation of a sphere of 1117 flexible TIP3P water molecules for switching intervals of two different lengths. Each of these simulations used a MTS scheme with a short time step of $0.7 \mathrm{fs}$ and a long time step of 2.8 fs. The upper graph shows the effect on energy conservation of a switch from 7.5 to $8.5 \AA$, whereas the lower graph shows the effect of a switch from 8.0 to $8.5 \AA$. As can be seen, the use of too short "healing" distances creates "slow" forces that are too fast and this makes energy conservation go from good to bad.

\section{Difficulties with FMA}

The combination of the Verlet-I/r-RESPA impulse method with the FMA was first studied in ref. 22. Good energy conservation was obtained with only four terms in the multipole expansion for long time steps $\Delta t$ as large as 4 or $4.5 \mathrm{fs}$. The largest system tested was the photosynthetic reaction center with 9513 atoms. In subsequent work with (rigid) SRC water and solvated proteins, ${ }^{39}$ six multipole terms we needed to maintain energy conservation.

Experiments are described here for the same solvated protein-DNA system, which suggest difficulties with even six multipole terms. A dynamics simulation was performed using a 1-fs time step for both the fast and slow forces so that the switching does not effect the results. Four levels of FMA cells were used, and all calculations were done in double precision. The results are given in Fig. 4, and they show that the use of six terms leads to an energy drift whereas the use of eight terms does not. It does not seem to be a simple 

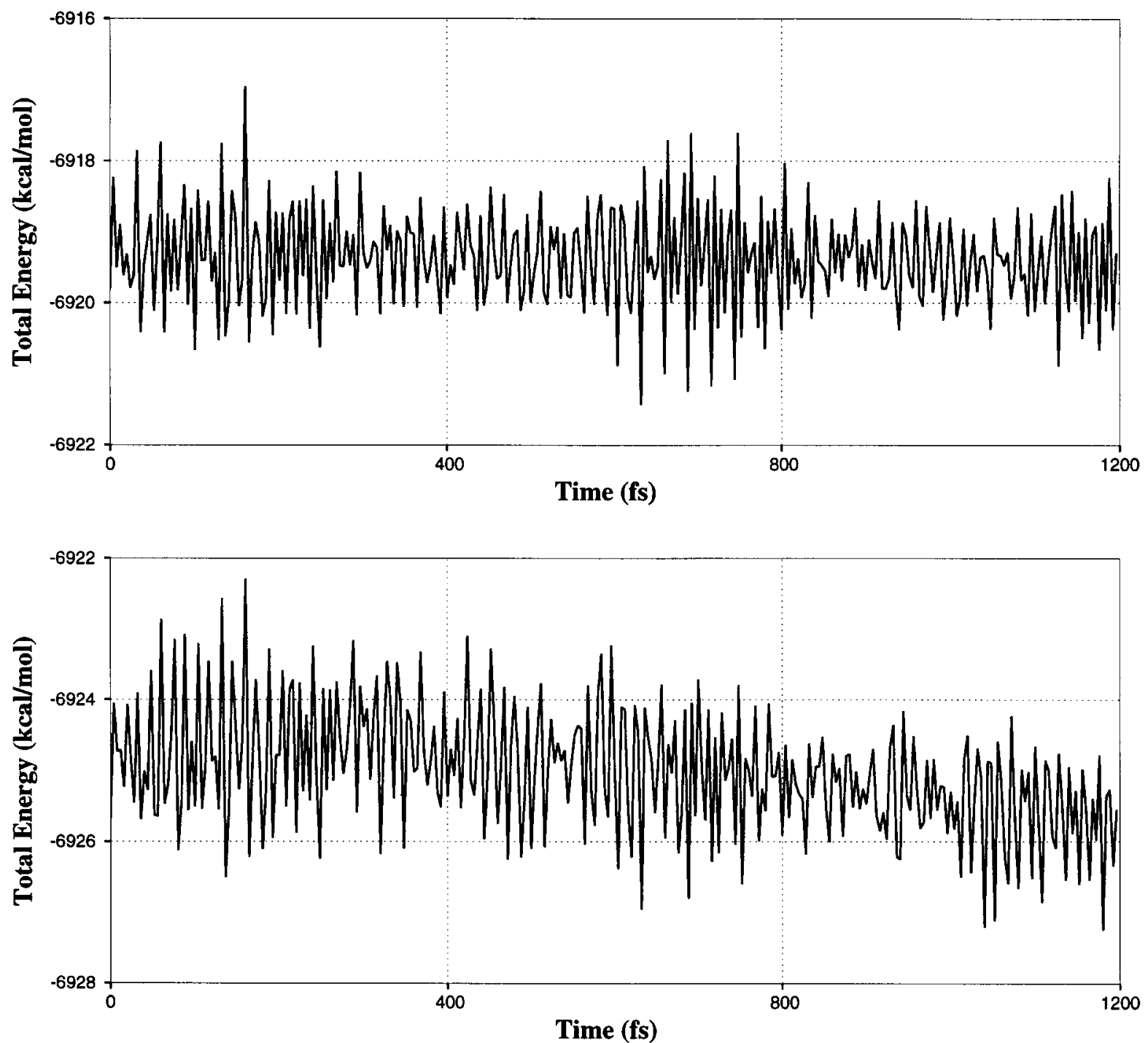

FIGURE 3. Drift in the energy due to the use of a too short healing distance for a simulation of a sphere of 1117 flexible TIP3P water molecules.

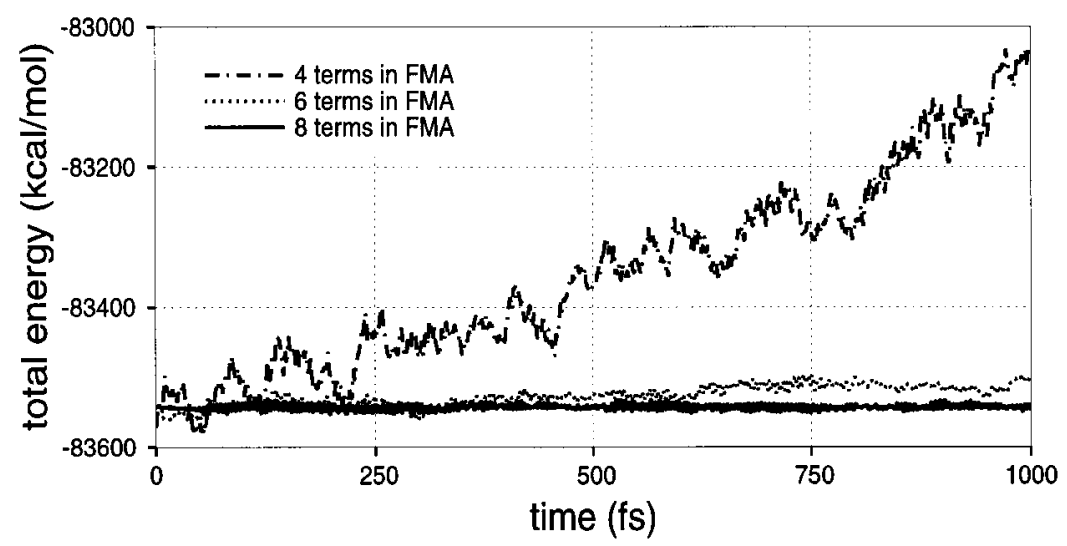

FIGURE 4. Energy conservation for the 36,000 -atom protein-DNA system using DPMTA multipole expansions of four, six, and eight terms at every time step. 
TABLE 1. DPMTA Calculation of Electrostatic Energy

\begin{tabular}{ll}
\hline No. terms & Relative Error \\
\hline 8 & $1.8 \times 10^{-5}$ \\
6 & $8.6 \times 10^{-5}$ \\
4 & $1.7 \times 10^{-4}$ \\
\hline
\end{tabular}

matter of inaccuracy. Table I gives the relative error in the DPMTA calculation of the electrostatic energy as a function of the number of terms; the six-term expansion appears to give more than enough accuracy. (Additional data on the accuracy of the FMA is given in ref. 40.) The data indicate that high accuracy is needed for energy conservation with the FMA. The simulation reported here differs from those cited above in that there are a large number of flexible covalent $\mathrm{H}$ bonds. For this sytem the (eight-processor) CPU time for computing an eight-term FMA long-range force is six times that required for computing the short-range forces.

The truncation of the multipole expansion yields a potential energy that is discontinuous as a function of the positions of the charged particles. In particular, there is a small jump in energy whenever a particle moves from one FMA cell to another. Numerical experiments indicate that small enough discontinuities can be tolerated. Note that extremely small discontinuities are undetectable due to the quantization of phase space induced by the use of finite precision numbers.

\section{Acknowledgment}

We are grateful to Dorina Kosztin for assistance with numerical experiments. The work was supported in part by the Beckman Institute for Advanced Science and Technology, NSF Grant BIR9318159, NIH Grant P41RR05969, and NSF Grant DMS-9600088.

\section{References}

1. R. J. Loncharich and B. R. Brooks, Proteins: Struct. Funct. Genet. 6, 32 (1989).

2. A. Warshel, in Modelling of Molecular Structures and Properties, J.-L. Rivail, Ed., Elsevier Science Publishers, Amsterdam, 1990, p. 515.
3. H. Grubmüller, H. Heller, A. Windemuth, and K. Schulten, Mol. Simul. 6, 121 (1991).

4. D. M. York, T. A. Darden, and L. G. Pedersen, J. Chem. Phys. 99, 8345 (1993).

5. M. Saito, J. Chem. Phys., 101, 1994

6. D. M. York, W. T. Yang, H. Lee, T. A. Darden, and L. G. Pedersen. J. Am. Chem. Soc., 117, 5001 (1995).

7. T. C. Bishop, D. Kosztin, and K. Schulten, Biophys. J., 72 2056 (1997).

8. D. Kosztin, T. C. Bishop, and K. Schulten, Biophys. J., 75 (1997).

9. M. Nelson, W. Humphrey, A. Gursoy, A. Dalke, L. Kalé, R. Skeel, K. Schulten, and R. Kufrin, Comput. Phys. Commun., 91, 111 (1995).

10. M. Nelson, W. Humphrey, A. Gursoy, A. Dalke, L. Kalé, R. D. Skeel, and K. Schulten, J. Supercomput. Appl. High Performance Comput., 10, 251 (1996).

11. A. Hayli, Bull. Astronom. 2, 67 (1967).

12. W. B. Street, D. J. Tildesley, and G. Saville, Mol. Phys. 35, 639 (1978).

13. W. F. van Gunsteren and H. J. C. Berendsen, GROMOS Manual, BIOMOS b. v., Phys. Chem. Lab., Univ. of Gronigen, 1987.

14. F. S. Lee and A. Warshel, J. Chem. Phys. 97, 3100 (1992).

15. H. Grubmüller, Master's thesis, Univ. München, Munich, 1989.

16. M. Tuckerman, B. J. Berne, and G. J. Martyna, J. Chem. Phys, 97, 1990 (1992).

17. J. J. Biesiadecki and R. D. Skeel, J. Comput. Phys., 109, 318 (1993).

18. J. M. Sanz-Serna and M. P. Calvo, Numerical Hamiltonian Problems, Chapman and Hall, London, 1994.

19. R. D. Skeel and J. J. Biesiadecki, Ann. Numer Math. 1, 191 (1994).

20. D. D. Humphreys, R. A. Friesner, and B. J. Berne, J. Phys. Chem., 98, 6885 (1994).

21. P. Procacci and M. Marchi, J. Chem. Phys., 104, 3003 (1996).

22. R. Zhou and B. J. Berne, J. Chem. Phys., 103, 9444 (1995).

23. L. Greengard and V. Rokhlin, J. Comput. Phys., 73, 325 (1987).

24. L. Greengard, The Rapid Evaluation of Potential Fields in Particle Systems, MIT Press, Cambridge, MA, 1988.

25. A. W. Appel, SIAM J. Sci. Stat. Comput., 6, 85 (1985).

26. J. Barnes and P. Hut, Nature, 324, 446 (1986).

27. Office of Science and Technology Policy, Computing Research News, September 8, 5, (1989).

28. K. E. Schmidt and M. A. Lee, J. Stat. Phys. 63, 1223 (1991).

29. Y.-S. Hwang, R. Das, J. H. Saltz, M. Hodošček, and B. R. Brooks IEEE Comput. Sci. Eng. 2, 18 (1995).

30. J. A. Board, Jr., Z. S. Hakura, W. D. Eilliot, D. C. Gray, W. J. Blanke, and J. F. Leathrum, Jr., In Proceedings of the Scalable High-Performance Computing Conference, Los Alamitos, CA IEEE Computer Society Press, New York, 1994, p. 87.

31. T. Hrycak and V. Rokhlin, SIAM J. Sci. Comput., to appear. [Yale Univ. Technical Report YALEU/DCS/RR-1089, November 27, 1995.] 
32. A. Windemuth, In Parallel Computing in Computational Chemistry, T. G. Mattson, Ed., ACS Books, Washington, D.C., 1995.

33. J. Board, Z. Hakura, W. Elliot, and W. Rankin, Scalable variants of multipole-accelerated algorithms for molecular dynamics applications, Technical Report TR94-006, Duke University Dept. of ELec. Eng., 1994.

34. T. Forester and W. Smith, Mol. Simul., 13, 195 (1994).

35. J. Wisdom, Astron. J., 87, 577 (1982).

36. H. Grubmüller, Ph.D. thesis, Univ. München, Munich, 1994.
37. H. Grubmüller and P. Tavan, Unpublished manuscript, 1994.

38. B. García-Archilla, J. M. Sanz-Serna, and R. D. Skeel, SIAM J. Sci. Comput., to appear. [Tech. Rept. 1996/7, Dept. Math. Appl. Comput., Univ. Valladolid, Valladolid, Spain.]

39. F. Figueirido, R. M. Levy, R. Zhou, and B. J. Berne, J. Chem. Phys., 106, 9835 (1997).

40. J. A. Board, Fr., J. W. Causey, J. F. Leathrum, Jr., A. WIndemuth, and K. Schulten, Chem. Phys. Lett., 198, 89 (1992). 